INGENIERIA INDUSTRIAL

\title{
Optimización multiobjetivo del problema de distribución de planta: Un nuevo modelo matemático
}

INDUSTRIAL ENGINEERING

\section{Multiobjective optimization of facility layout problem: A new mathematical model}

\author{
Alfredo D. Moreno*§, Aldair A. Álvarez*, Víctor M. Noble*, Jorge M. López* \\ *Departamento de ingeniería Industrial, Universidad de Córdoba, Montería, Colombia. \\ §alfredmorenoarteaga@gmail.com,aaad_147@hotmail.com,vicmanr90@gmail.com,jotamlopez@ \\ gmail.com
}

Recibido: Septiembre 4 de 2013 - Aceptado: Julio 15 de 2014

\begin{abstract}
Resumen
Este artículo presenta un nuevo modelo matemático multiobjetivo para la optimización del problema de distribución de instalaciones de áreas desiguales conocido como UA-FLP de dimensiones fijas. Uno de los objetivos es la minimización de los costos de manejo de materiales y el otro es la minimización de las relaciones de cercanía basadas en criterios independientes del flujo y el costo entre estaciones de trabajo. Para la validación del modelo matemático se utilizan diferentes instancias de problemas encontrados en la literatura. Como resultado se encuentra que el modelo matemático propuesto representa fielmente el problema de distribución de instalaciones de dimensiones fijas y áreas desiguales optimizando el costo de manejo de materiales y las relaciones de cercanía para problemas pequeños y proporcionando soluciones de buena calidad para problemas de mayor tamaño en un tiempo computacional de 10,000 segundos.
\end{abstract}

Palabras claves: Distribución de instalaciones, manejo de materiales, modelo matemático, optimización multiobjetivo, relaciones de cercanía.

\begin{abstract}
This paper presents a new multiobjetive mathematical model for optimization of facility layout problem with unequal areas called UA-FLP with fixed dimensions. One of the objectives is the minimization of the material handling cost and the other one is the minimization of the closeness relationships according to factors that are independents of the flows and costs. The validation of the mathematical model was made with problems found in the literature. The result was that the proposed model represents the unequal area facility layout problem with fixed dimensions optimizing the material handling cost and the closeness relationships for small problems and finding good solutions for bigger problems in 10,000 seconds of computer time.
\end{abstract}

Keywords: Closeness relationships, facility layout, material handling, mathematical model, multiobjetive optimization. 


\section{Introducción}

Son muchas las problemáticas a las que se enfrentan las organizaciones en la actualidad, lo que ha obligado a las empresas a optimizar sus recursos y buscar métodos que le permitan ser competitivas y mantenerse rentables en el tiempo. La distribución de planta es un factor que sin duda contribuye a mejorar la rentabilidad, por lo que se hace necesario el diseño de modelos que representen fielmente el problema y que puedan ser utilizados para obtener buenas soluciones en tiempos computacionales aceptables. Existen diferentes tipos de problemas de distribución de instalaciones entre ellos el problema de distribución de instalaciones con áreas desiguales o UA-FLP (Unequal área layout problem) en el que al menos una de las instalaciones tiene un área diferente a otra (Garci, Pierreval, SalasMorera, \& Arauzo-Azofra, 2010).

Para realizar una adecuada distribución de instalaciones en los sistemas productivos las organizaciones deben tener en cuenta, además de los costos por manejo de materiales, factores como la organización lógica y secuencial de los sistemas de producción, la seguridad de las personas, los materiales y la maquinaria, la minimización de la distancia entre actividades en las que se manejen materiales peligrosos y las relaciones de adyacencia o separación por razones de seguridad, ruido o limpieza. El principal problema que se presenta al querer tener en cuenta todos estos factores es que en la literatura son pocos los modelos que tiene en cuenta aspectos de importancia de cercanía independientes del costo de manejo de materiales. Por lo tanto, existe la necesidad de la construcción de modelos matemáticos que representen, y sirvan para resolver de forma exacta, el FLP de áreas desiguales y dimensiones fijas y que tengan como objetivo tanto la optimización del costo de manejo de materiales entre estaciones de trabajo como la optimización de las relaciones de cercanías entre estaciones basadas en criterios independientes de los flujos y los costos entre estaciones.

\subsection{El problema de distribución de planta (FLP)}

La distribución en planta es definida como la integración de arreglos físicos como máquinas, materiales, departamentos, estaciones de trabajo, áreas de almacenamiento y otras áreas comunes buscando la producción de productos de la manera más eficiente Maniya \& Bhatt (2011). El problema consiste en realizar la integración de todos estos arreglos físicos y distribuir de manera óptima todos los departamentos que intervengan en un proceso productivo. Pretende conseguir el objetivo de ubicar distintos recursos o departamentos dentro de una superficie, a fin de lograr la mayor eficiencia en la producción de un bien o servicio Tompkins \& White (1984). El FLP es conocido por ser NP-hard (Ohmori, Yoshimoto, \& Ogawa, 2010), lo que implica que en general es difícil de resolver.

\subsection{Modelos que representan el problema de distribución de planta}

El problema de distribución de instalaciones (FLP) se encuentra en la literatura representado principalmente a través de modelos QAP (Quadratic Assignment Problem) y MIP (Mixed Integer Programming) Drira et al. (2007). En el problema de asignación cuadrática (QAP) el plano o planta donde se va a realizar la distribución es dividida en bloques rectangulares con la misma área y forma y los bloques son asignados a instalaciones para llevar a cabo la distribución. Si las instalaciones o departamentos tienen áreas desiguales, estas pueden ocupar diferente número de bloques o espacios Sadrzadeh (2012). En el problema de programación entera mixta (MIP Problem) todas las instalaciones son ubicadas en un lugar dentro del sitio designado para ello y no debe haber solapamiento entre ellas. Las instalaciones en la planta son localizadas por las coordenadas de sus centroides y sus dimensiones o por las coordenadas de su punto inferior izquierdo y sus dimensiones Drira et al. (2007).

En la tabla 1 se muestra la recopilación de diversas técnicas de modelación en diferentes referencias. 
Tabla 1. Principales técnicas de modelación matemática encontradas en la literatura Fuente: Kundu \& Dan (2012)

\begin{tabular}{|c|c|}
\hline Referencia & $\begin{array}{c}\text { Técnica de modelación } \\
\text { matemática }\end{array}$ \\
\hline Castillo \& Peters (2003) & NLMIP \\
\hline Chiang (2001) & $Q A P$ \\
\hline Dong et al. (2009) & $M I P$ \\
\hline Ioannou (2007) & $I P$ \\
\hline Matsuzaki et al. (1999) & QAP and slicing tree \\
\hline McKendall et al. (2006) & $Q A P$ \\
\hline Mir \& Imam (2001) & MIP \\
\hline Sahin \& Türkbey (2009) & $Q A P$ \\
\hline Singh \& Sharma (2007) & $Q A P$ \\
\hline Sugiyono (2006) & Bond energy algorithm \\
\hline Yang et al. (2005) & $M I P$ \\
\hline $\begin{array}{c}\text { McKendall \& Hakobyan } \\
\text { (2010) }\end{array}$ & MILP \\
\hline Scholz et al. (2009) & $Q A P$ \\
\hline Seo et al. (2006) & $Q A P$ \\
\hline Delmaire et al. (1997) & $L P$ \\
\hline Diego-Mas et al. (2009) & Slicing Tree \\
\hline Dunker et al. (2005) & $D P$ \\
\hline Eklund et al. (2006) & MIP \\
\hline Gau \& Meller (1999) & $M I P$ \\
\hline Hicks (2004) & Agglomerative clustering \\
\hline$H u \&$ Wang (2004) & $Q A P$ \\
\hline Kochhar \& Heragu (1999) & MIP \\
\hline $\begin{array}{c}\text { Kulkarni \& Shanker } \\
\text { (2007) }\end{array}$ & $Q A P$ \\
\hline Liu \& Li (2006) & $M I P$ \\
\hline Longo et al. (2005) & $Q A P$ \\
\hline Osman et al. (2003) & $Q A P$ \\
\hline Rajasekharan et al. (1998) & MIP \\
\hline Ramkumar et al. (2009) & $Q A P$ \\
\hline Wu \& Appleton (2002) & $Q A P$ \\
\hline Wu et al. (2007) & $M I P$ \\
\hline Hani et al. (2007) & $Q A P$ \\
\hline Hardin \& Usher (2005) & $Q A P$ \\
\hline Paul et al. (2006) & $Q A P$ \\
\hline Solimanpur et al. (2004) & $Q A P$ \\
\hline $\begin{array}{c}\text { Teo \& Ponnambalam } \\
\text { (2008) }\end{array}$ & $N L P$ \\
\hline
\end{tabular}

\section{Metodología}

\subsection{Formulación del nuevo modelo matemático}

Salas-Morera et al. (2011) establecen que una buena distribución en planta es aquella que tiene en cuenta aspectos que pueden ser cuantificados como el costo de manejo de materiales, la relación lógica entre estaciones y la forma de cada área.

El costo de manejo de materiales puede ser calculado a partir de la Ec. (1):

$$
F_{1}=\sum_{i=1}^{n} \sum_{j=1}^{n} d_{i j} * c_{i j} * f_{i j}
$$

Dónde: $i, j$ corresponden al conjunto de instalaciones que deben ubicarse; $\mathrm{n}$ es el número total de estaciones o instalaciones a distribuir; $c_{i j}$ es el costo de transporte entre la estación $i$ y la estación $j$ por unidad de flujo por unidad de distancia; $f_{i j}$ es el flujo de productos entre la estación $i$ y la estación $j$ y $d_{i j}$ es la distancia total rectilínea que existe entre los centroides de las estaciones $i$ y $j$.

La matriz de costos $(c)$ y flujos $(f)$ se determinan de acuerdo a valores cuantitativos $y$, muchas veces medibles, que tienen las empresas. La matriz de costos indica lo que cuesta transportar materiales o productos de una estación a otra. La matriz de flujo depende, como su nombre lo indica, de la cantidad de flujo de material o productos que existe entre las estaciones.

Por otro lado, la relación lógica entre estaciones depende de los ratios lógicos de importancia. Los ratios de importancia o de cercanía entre estaciones pueden ser establecidos de acuerdo a diferentes criterios tales como, de acuerdo a Salas-Morera et al. (2011), la organización lógica y secuencial de los sistemas de producción, la ausencia o presencia de ruido y la seguridad de las personas, los materiales y la maquinaria. Shah et al. (2010) establecen que la importancia entre instalaciones se debe determinar de acuerdo a reducir las distancias entre actividades en las que se manejen materiales peligrosos, relaciones de 
adyacencia o separación por razones de seguridad, ruido o limpieza.

Algunos autores establecen estos ratios de importancia de acuerdo a los flujos entre estaciones, lo cual es algo redundante, aunque no es necesariamente un problema al momento de realizar la distribución (Salas-Morera et al., 2011).

Para tener en cuenta la relación lógica entre estaciones se utiliza la Ec.(2):

$$
F_{2}=\sum_{i=1}^{n} \sum_{j=1}^{n} d_{i j} * R E L_{i j}
$$

Donde: $\left(R E L_{i j}\right)$ es el ratio de importancia o relación de cercanía entre la estación $i$ y la estación $j$.

Los ratios de cercanía entre estaciones $\left(R E L_{i j}\right)$ dependen de la relación lógica existente entre cada par de instalaciones $i j$ y pueden ser establecidos de acuerdo a las características del problema. Se basan en los siguientes valores: Relaciones esenciales (Essential closeness) con una valor de 410, Relaciones muy importantes (Very Important Closeness) con 130, relaciones importantes (Important Closeness) con 50, relaciones normales (Normal Closeness) con 20, relaciones indiferentes (Indifferent Closeness) con 10 y relaciones indeseables (Undesirable Closeness) con 0.

En la literatura no se cuenta con instancias que tengan matriz de costos por flujo y matriz de relaciones de importancia simultáneamente para el problema de distribución de instalaciones de áreas desiguales y dimensiones fijas. Por esta razón para este trabajo el cálculo de los ratios de importancia se realizó de manera aleatoria dando una probabilidad de 1/6 a cada valor de ratios de distancias.

Para representar las funciones objetivos a través de una única función que considere los aspectos de costo de manejo de materiales y relaciones de cercanía se utiliza el valor $(\alpha)$ que representa la importancia de cada uno de los objetivos en la toma de decisiones. La función objetivo queda representada entonces de la siguiente manera:

$$
\begin{gathered}
F .0 .=\min Z=\propto *\left(F_{1}=\sum_{i=1}^{n} \sum_{j=1}^{n} d_{i j} * c_{i j} * f_{i j}\right)+ \\
(1-\propto) *\left(\sum_{i=1}^{n} \sum_{j=1}^{n} d_{i j} * r e l_{i j}\right)
\end{gathered}
$$

Dónde: $\alpha$ es un factor de ponderación que puede tomar valores entre 0 y $1 ; \mathrm{Z}$ es el valor total de la función objetivo, considerada como una función de costos que debe ser minimizada y que lleva en cuenta el costo de manejo de materiales y la importancia de la relación entre estaciones.

$$
d_{i j}=\left|X_{i}-X_{j}\right|+\left|Y_{i}-Y_{j}\right|
$$

Para el cálculo de la distancia se utilizara la métrica Minkowski $\mathrm{p}=1$ (Manhattan) (Tompkins \& White, 1984) y se rige por la siguiente fórmula:

$$
\begin{gathered}
\mathrm{dx}_{\mathrm{ij}} \geq \mathrm{X}_{\mathrm{i}}+\left(\frac{\left(\left(1-\mathrm{P}_{\mathrm{i}}\right) * \mathrm{w}_{\mathrm{i}}+\left(\mathrm{P}_{\mathrm{i}}\right) * \mathrm{~h}_{\mathrm{i}}\right)}{2}\right)- \\
\mathrm{X}_{\mathrm{j}}-\left(\frac{\left(\left(1-\mathrm{P}_{\mathrm{j}}\right) * \mathrm{w}_{\mathrm{j}}+\left(\mathrm{P}_{\mathrm{j}}\right) * \mathrm{~h}_{\mathrm{j}}\right)}{2}\right) \\
\mathrm{dx}_{\mathrm{ij}} \geq \mathrm{X}_{\mathrm{j}}+\left(\frac{\left(\left(1-\mathrm{P}_{\mathrm{j}}\right) * \mathrm{w}_{\mathrm{j}}+\left(\mathrm{P}_{\mathrm{j}}\right) * \mathrm{~h}_{\mathrm{j}}\right)}{2}\right)- \\
\mathrm{X}_{\mathrm{i}}-\left(\frac{\left(\left(1-\mathrm{P}_{\mathrm{i}}\right) * \mathrm{w}_{\mathrm{i}}+\left(\mathrm{P}_{\mathrm{i}}\right) * \mathrm{~h}_{\mathrm{i}}\right)}{2}\right) \\
\mathrm{dy}_{\mathrm{ij}} \geq \mathrm{Y}_{\mathrm{i}}+\left(\frac{\left(\left(1-\mathrm{P}_{\mathrm{i}}\right) * \mathrm{~h}_{\mathrm{i}}+\left(\mathrm{P}_{\mathrm{i}}\right) * \mathrm{w}_{\mathrm{i}}\right)}{2}\right)- \\
\mathrm{Y}_{\mathrm{j}}-\left(\frac{\left(\left(1-\mathrm{P}_{\mathrm{j}}\right) * \mathrm{~h}_{\mathrm{j}}+\left(\mathrm{P}_{\mathrm{j}}\right) * \mathrm{w}_{\mathrm{j}}\right)}{2}\right) \\
\mathrm{dy}_{\mathrm{ij}} \geq \mathrm{Y}_{\mathrm{j}}+\left(\frac{\left(\left(1-\mathrm{P}_{\mathrm{j}}\right) * \mathrm{~h}_{\mathrm{j}}+\left(\mathrm{P}_{\mathrm{j}}\right) * \mathrm{w}_{\mathrm{j}}\right)}{2}\right)- \\
\mathrm{Y}_{\mathrm{i}}-\left(\frac{\left(\left(1-\mathrm{P}_{\mathrm{i}}\right) * \mathrm{~h}_{\mathrm{i}}+\left(\mathrm{P}_{\mathrm{i}}\right) * \mathrm{w}_{\mathrm{i}}\right)}{2}\right) \\
d_{i j}=d x_{i j}+d y_{i j}
\end{gathered}
$$


Donde: $h_{i}$ es la medida del alto de la estación $i ; w$ es la medida del ancho de la estación $i ; P i$ es una variable binaria que toma el valor de 0 cuando la dimensión en $x$ de la estación $i$ es igual al ancho wi y el valor de 1 cuando la dimensión en $\mathrm{x}$ de la estación $i$ es igual al alto $h_{i} ; d x_{i j}$ es la distancia horizontal que existe entre los centroides de las estaciones $i$ y $j$; $d y_{i j}$ es la distancia vertical que existe entre los centroides de las estaciones $i \mathrm{y} j ; X_{i}$ es la coordenada en $X$ del punto inferior izquierdo de la estación $i$; $Y_{i}$ es la coordenada en $Y$ del punto inferior izquierdo de la estación $i$.

La Ec. (5) y Ec. (6) se utilizan para encontrar la distancia horizontal entre los centroides de las estaciones $i$ y $j$. La Ec. (7) y Ec. (8) se utilizan para encontrar la distancia vertical entre los centroides de las estaciones $i$ y $j$. La Ec. (9) Se utiliza para encontrar la distancia rectilínea total (Manhattan) entre los centroides de las estaciones $i$ y $j$.

Para evitar el solapamiento entre estaciones se hace uso de cuatro variables binarias y cinco restricciones como se muestra a continuación:

$$
\begin{gathered}
B *\left(L_{i j}\right) \leq X_{i}-X_{j}-\left(1-P_{j}\right) * w_{j}-\left(P_{j}\right) * h_{j} \forall i \neq j(10) \\
B *\left(M_{i j}\right) \leq Y_{i}-Y_{j}-\left(1-P_{j}\right) * h_{j}-\left(P_{j}\right) * w_{j} \forall i \neq j(11) \\
B *\left(Q_{i j}\right) \leq X_{j}-X_{i}-\left(1-P_{i}\right) * w_{i}-\left(P_{i}\right) * h_{i} \forall i \neq j(12) \\
B *\left(T_{i j}\right) \leq Y_{j}-Y_{i}-\left(1-P_{i}\right) * h_{i}-\left(P_{i}\right) * w_{i} \forall i \neq j \\
L_{i j}+M_{i j}+Q_{i j}+T_{i j} \geq 1 \forall i \neq j
\end{gathered}
$$

Dónde: B es definida como una constante muy grande y es igual a la sumatoria del máximo entre el ancho y el alto de las estaciones $(B=$ $\left.\sum_{i} \max \left\{w_{i}, h_{i}\right\}\right)$, esto garantiza que en el plano se puedan generar todas las posibles soluciones de la distribución; $L_{i j}$ es una variable binaria que toma el valor de 1 cuando la instalación $i$ está a la derecha de la instalación $j$ y 0 en caso contrario; $M_{i j}$ es una variable binaria que toma el valor de 1 cuando la instalación $i$ está por encima de la instalación $j$ y 0 en caso contrario; $Q_{i j}$ es una variable binaria que toma el valor de 1 cuando la instalación $i$ está a la izquierda de la instalación $j$ y 0 en caso contrario; $T_{i j}$ es una variable binaria que toma el valor de
1 cuando la instalación $i$ está por debajo de la instalación $j$ y 0 en caso contrario.

La Ec. (10), Ec. (11), Ec. (12), Ec. (13) y Ec. (14) son restricción de solapamiento que se utilizan para impedir que dos estaciones diferentes tengan puntos ubicados en el mismo lugar en el plano. Una estación $i$ no está solapada con una estación $j$, solo si cumple uno o más de los siguientes casos: La estación i está ubicada a la derecha de la estación $\mathrm{j}$, en este caso la variable binaria $L_{i j}$ de la Ec. (10) toma el valor de uno; La estación $i$ está a la izquierda de la estación $j$, en este caso la variable binaria $M_{i j}$ de la Ec. (11) toma el valor de uno; La estación $i$ está por arriba de la estación $j$, en este caso la variable binaria $Q_{i j}$ de la Ec. (12) toma el valor de uno; y la estación i está por debajo de la estación j, en este caso la variable binaria $T_{i j}$ de la Ec. (13) toma el valor de uno.

Cuando al menos una de estas condiciones se cumple entonces la suma de las variables binaria $L, M, Q$ y $T$ en la Ec.14 es igual a uno. Cuando más de una de estas condiciones se cumple entonces la suma de estas variables es mayor que uno.

El anterior modelo matemático fue inspirado en el modelo MILP propuesto por Papageorgiou \& Rotstein (1998).

\subsection{Instancias del problema}

En esta investigación se utilizan 17 instancias de problemas encontrados en la literatura que van desde problemas con 5 estaciones hasta problemas con 49 estaciones.

Las instancias utilizadas son tomadas de diferentes fuentes bibliográficas. Las instancias ami49, ami33, hp, Xerox y apte son tomadas de MCNC Benchmark Problems referenciadas Ohmori et al. (2010). Las instancias Example 1, Example 2, Example 3 y Example 4 son tomadas de Xu \& Papageorgiou (2009). Las instancias Nugent 5, $6,7,8,12,15,20$ y 30 son tomadas de Nugent et al. (1968). En estos problemas las estaciones son cuadradas de $1 \mathrm{X} 1$. 
Todos estos problemas proporcionan la matriz de costo por flujo y las dimensiones del alto y el ancho de las estaciones; pero, no muestran las relaciones de importancia entre departamentos, las cuales se generan de manera a aleatoria.

\subsection{Experimentos computacionales}

En total se realizaron 21 corridas para cada una de las 17 instancias utilizadas. Estas corridas se realizaron variando el alfa $(\alpha)$ en 0.05 desde 0 hasta 1.

Para cada una de las corridas se utiliza el software GAMS 22.6 y el solver CPLEX 11.0 con un límite para el tiempo de cómputo de 10000 segundos (10.000s CPU limit), que es el límite de recursos computacionales utilizado por Xu \& Papageorgiou (2009).

Los experimentos se realizan en PC con procesador Intel Corel Dual, $2 \mathrm{GHz}, 1$ GB de memoria RAM y sistema operativo Windows 7 de 32 bits.

\section{Resultados y discusión}

\subsection{Validación del modelo matemático}

Para realizar la validación del modelo matemático se toman instancias del problema de distribución en planta de áreas desiguales y dimensiones fijas encontrados en la literatura y se comparan los resultados. Sin embargo, dado que en la literatura no se encuentran problemas que aborden la optimización del costo de manejo de materiales y las relaciones de cercanía simultáneamente se tomara para esta parte solo la función de costos por manejo de materiales con el fin de poder comparar directamente los resultados del modelo propuesto con los casos encontrados en la literatura. Los resultados arrojados con la implementación del modelo se muestran en la tabla 1.

Para las 7 instancias utilizadas en la validación, como se puede observar en la tabla 2 , se encontró la solución óptima. Las soluciones óptimas encontradas con la aplicación del modelo coinciden con las soluciones óptimas encontradas en la literatura, lo que indica que el modelo es válido para representar el UA-FLP de dimensiones fijas. En la figura 1 se muestra la distribución arrojada por el modelo y la mejor encontrada en la literatura para una de estas instancias. Se observa que en todos los casos la distribución óptima del modelo es igual a la distribución óptima de la literatura.

\subsection{Resultados obtenidos para f1}

Al utilizar el valor de $\alpha=1$ se le atribuye todo el peso de la ecuación al objetivo de costo de manejo de materiales. En este caso se puede realizar una comparación con los mejores resultados para estos problemas encontrados en la literatura. La tabla 3 muestra el resultado para f1 cuando $\alpha=1$, el tiempo computacional empleado y la diferencia con la mejor solución encontrada para las 17 instancias estudiadas.

Tabla 2. Resultados óptimos de la implementación para problemas de la literatura.

\begin{tabular}{|c|c|c|c|c|c|}
\hline Instancia & $A$ & Número de departamentos & Tiempo (s) & F.O & Mejor F.O \\
\hline Example 1 & 1 & 7 & 1,78 & $9.948,03$ & $\begin{array}{c}9.948,03 \text { en Xu \& } \\
\text { Papageorgiou }(2009)\end{array}$ \\
\hline Example 2 & 1 & 12 & 359,65 & $17.190,00$ & $\begin{array}{c}\text { 17.190,00 en Xu \& } \\
\text { Papageorgiou (2009) }\end{array}$ \\
\hline Example 3 & 1 & 18 & $>10000$ & $31.640,00$ & $\begin{array}{c}\text { 31.640,00 en Xu \& } \\
\text { Papageorgiou (2009) }\end{array}$ \\
\hline Nugent 5 & 1 & 5 & 0,23 & 50,00 & 50,00 en Chiang (2001) \\
\hline Nugent 6 & 1 & 6 & 1,12 & 86,00 & 86,00 en Chiang (2001) \\
\hline Nugent 7 & 1 & 7 & 23,37 & 144,00 & $\begin{array}{c}\text { 144,00 en Chiang (2001) } \\
\text { (Chiang, 2001) }\end{array}$ \\
\hline Nugent 8 & 1 & 8 & 432,84 & 212,00 & 212,00 en Chiang (2001) \\
\hline
\end{tabular}




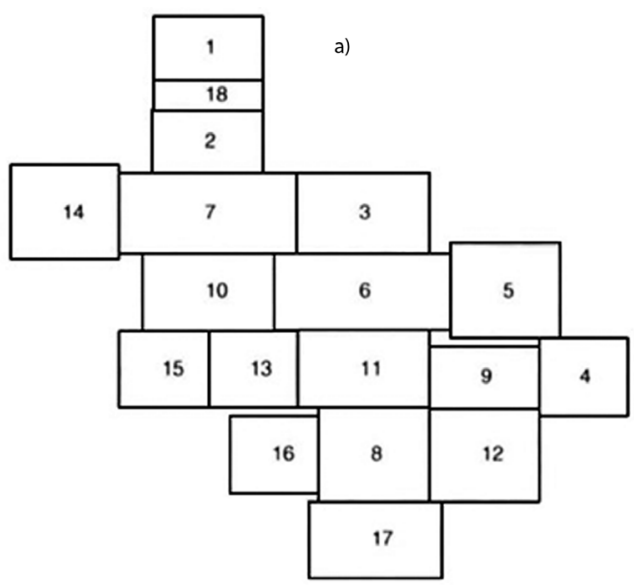

b)

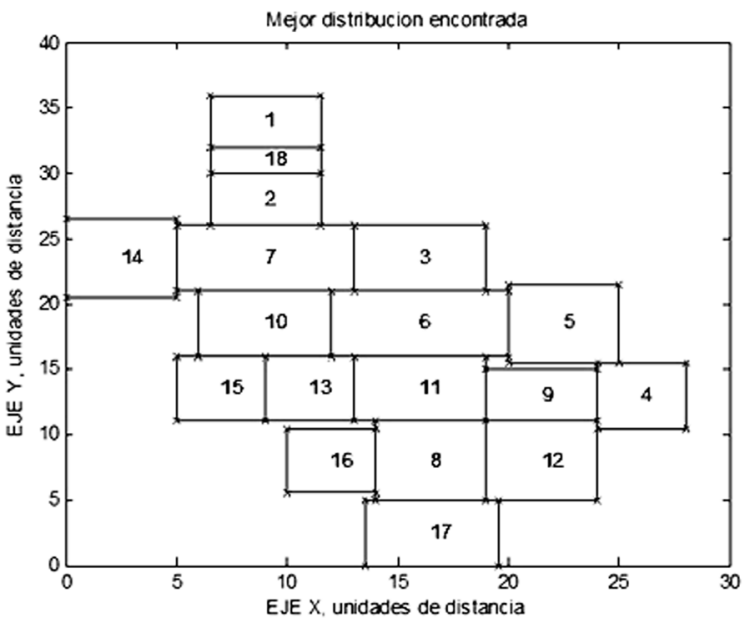

Figura 1. a) Solución óptima para ejemplo 3 de acuerdo a Xu \& Papageorgiou (2009), y b) Solución óptima para ejemplo 2 de acuerdo al modelo propuesto.

Los resultados de las instancias Apte, Xerox, Hp, Ami33 y Ami49 se comparan con los resultados obtenidos en Ohmori et al. (2010). Los autores utilizan las metaheurísticas nube de partículas (PSO) y recocido simulado (SA) para darle solución a estas instancias. Como se puede observar en la tabla 3, la utilización del modelo mejoró los resultados de la metaheurísticas PSO y SA para cuatro de las 5 instancias utilizadas de MCNC Benchmark Problems en un tiempo de cómputo de 10,000 segundos.

En cuanto a los Example Problems se tiene que para los problemas Example 1 y 2 se encontraron soluciones óptimas, y buenas soluciones para los problemas Example 3 y 4 en menos de 10,000 segundos. Estos resultados son comparados con los obtenidos en $\mathrm{Xu} \&$ Papageorgiou (2009), donde los autores utilizan un modelo llamado Layout con el que dentro del límite de 10,000 segundos solo encuentran la solución óptima para la instancia Example 1. Luego diseñan un algoritmo de mejora que les permite encontrar mejores soluciones en menos tiempo (4.09 segundos para Example 2). El modelo propuesto en este trabajo por si solo mejora la eficiencia en comparación con el modelo Layout, aunque es menos eficiente que el algoritmo de mejora que proponen los autores en $\mathrm{Xu} \&$ Papageorgiou (2009).

Para los Nugent Problems se encontraron soluciones óptimas en menos de 10,000 segundos para Nugent 5, 6, 7 y 8 y buenas soluciones para las instancias Nugent 12, 15, 20 y 30. Estas instancias son resueltas en Chiang (2001) empleando un algoritmo de búsqueda Tabu y una heurística de control de vecindario. Los resultados muestran que el modelo propuesto por si solo es menos eficiente que la metodología empleada en Chiang (2001) para encontrar buenas soluciones para problemas de gran tamaño.

Como se puede observar, la aplicación del modelo para resolver problemas de distribución de planta de áreas desiguales y dimensiones fijas es eficiente desde el punto de vista del tiempo computacional y la calidad de respuesta para problemas con menos de 8 estaciones arrojando resultados óptimos en poco tiempo. Para problemas de más de 8 instalaciones en 10,000 segundos se encuentran soluciones que se alejan en promedio un $13 \%$ de la mejor solución encontrada.

\subsection{Resultados multiobjetivo}

Para cada una de las instancias se realizaron 21 corridas variando el valor del $\alpha$ en 0.05 de 0 a 1 . Una vez obtenidos los valores para cada una de estas corridas se seleccionan las soluciones no dominadas y se construye el frente de Pareto. En la figura 2 se puede observar la gráfica de todos los resultados obtenidos y la gráfica de frente de Pareto con las soluciones no dominadas para la instancia Xerox. 
Tabla 3. Resultados de la implementación del modelo para problemas con soluciones en la literatura.

\begin{tabular}{|c|c|c|c|c|c|c|c|}
\hline Instancia & $\begin{array}{c}\text { Número de } \\
\text { departamentos }\end{array}$ & $\begin{array}{l}\text { Tiempo } \\
\text { (s) }\end{array}$ & F.O $(\alpha=1)$ & Mejor f.O & \% Diferencia & $F . O(\alpha=0,05)$ & \% Diferencia \\
\hline Apte & 9 & 10.000 & $248.744,37$ & $\begin{array}{l}\text { 261.001,60 en } \\
\text { (Ohmori et al., } \\
\text { 2010) }\end{array}$ & $-4,70 \%$ & $329.608,00$ & $26,29 \%$ \\
\hline Xerox & 10 & 10.000 & $187.998,96$ & $\begin{array}{l}208.644,70 \text { en } \\
\text { (Ohmori et al., } \\
\text { 2010) }\end{array}$ & $-9,90 \%$ & $259.588,00$ & $24,42 \%$ \\
\hline$H p$ & 11 & 10.000 & $123.585,09$ & $\begin{array}{l}151.772,30 \text { en } \\
\text { (Ohmori et al., } \\
\text { 2010) }\end{array}$ & $-18,57 \%$ & $162.484,00$ & $7,06 \%$ \\
\hline Ami33 & 33 & 10.000 & $654.871,25$ & $\begin{array}{c}728.011,50 \text { en } \\
\text { (Ohmori et al., } \\
\text { 2010) }\end{array}$ & $-10,05 \%$ & $776.104,00$ & $6,61 \%$ \\
\hline Ami49 & 49 & 10.000 & $8.834 .644,29$ & $\begin{array}{c}\text { 8.169.529,30 en } \\
\text { (Ohmori et al., } \\
\text { 2010) }\end{array}$ & $8,14 \%$ & $9.261 .056,00$ & $13,36 \%$ \\
\hline Example 1 & 7 & 1,78 & $\begin{array}{l}9.948,03 \\
\text { (optimo) }\end{array}$ & $\begin{array}{l}9.948,03 \text { en (Xu } \\
\& \text { Papageorgiou, } \\
2009) 23.10 \mathrm{seg} .\end{array}$ & $0,00 \%$ & $13.795,62$ & $38,68 \%$ \\
\hline Example 2 & 12 & 359,65 & $\begin{array}{c}17.190,00 \\
\text { (optimo) }\end{array}$ & $\begin{array}{l}\text { 17.190,00 en }(X u \\
\& \text { Papageorgiou, } \\
2009) 4.09 \text { seg. }\end{array}$ & $0,00 \%$ & $22.440,00$ & $30,54 \%$ \\
\hline Example 3 & 18 & 10.000 & $33.312,50$ & $\begin{array}{l}\text { 31.640,00 en }(X u \\
\text { \& Papageorgiou, } \\
\text { 2009) 419,62 seg. }\end{array}$ & $5,29 \%$ & $33.312,50$ & $5,05 \%$ \\
\hline Example 4 & 36 & 10.000 & $5.062,26$ & $\begin{array}{c}\text { 3.873,81 en }(X u \\
\& \text { Papageorgiou, } \\
\text { 2009) } 6497.31 \\
\text { seg. }\end{array}$ & $30,68 \%$ & $69.247,50$ & $1687,58 \%$ \\
\hline Nugent 5 & 5 & 0,23 & $\begin{array}{c}50,00 \\
\text { (optimo) }\end{array}$ & $\begin{array}{l}\text { 50,00 en (Chiang, } \\
\text { 2001) } 1 \text { seg. }\end{array}$ & $0,00 \%$ & 50,00 & $0,00 \%$ \\
\hline Nugent 6 & 6 & 1,12 & $\begin{array}{c}86,00 \\
\text { (optimo) }\end{array}$ & $\begin{array}{c}\text { 86,00 en (Chiang, } \\
\text { 2001) } 1 \mathrm{seg}\end{array}$ & $0,00 \%$ & 108,00 & $25,58 \%$ \\
\hline Nugent 7 & 7 & 23,37 & $\begin{array}{c}144,00 \\
\text { (optimo) }\end{array}$ & $\begin{array}{l}\text { 144,00 en (Chiang, } \\
\text { 2001) } 2 \text { seg. }\end{array}$ & $0,00 \%$ & 156,00 & $8,33 \%$ \\
\hline Nugent 8 & 8 & 432,84 & $\begin{array}{l}212,00 \\
\text { (optimo) }\end{array}$ & $\begin{array}{l}\text { 212,00 en (Chiang, } \\
\text { 2001) } 2 \text { seg. }\end{array}$ & $0,00 \%$ & 286,00 & $34,91 \%$ \\
\hline Nugent 12 & 12 & 10.000 & 606,00 & $\begin{array}{l}\text { 578,00 en (Chiang, } \\
\text { 2001) } 80 \text { seg. }\end{array}$ & $4,84 \%$ & $3.840,00$ & $564,36 \%$ \\
\hline Nugent 15 & 15 & 10.000 & $1.246,00$ & $\begin{array}{c}1.110,00 \text { en } \\
\text { (Chiang, 2001) } 40 \\
\text { seg. }\end{array}$ & $12,25 \%$ & $1.662,00$ & $49,73 \%$ \\
\hline Nugent 20 & 20 & 10.000 & $2.948,00$ & $\begin{array}{c}\text { 2.564,00 en } \\
\text { (Chiang, 2001) } \\
\text { 1489 seg. }\end{array}$ & $14,98 \%$ & $3.530,00$ & $37,68 \%$ \\
\hline Nugent 30 & 30 & 10.000 & $6.972,00$ & $\begin{array}{c}\text { 6.094,00 en } \\
\text { (Chiang, 2001) } 65 \\
\text { seg. }\end{array}$ & $14,41 \%$ & $8.136,00$ & $33,51 \%$ \\
\hline
\end{tabular}



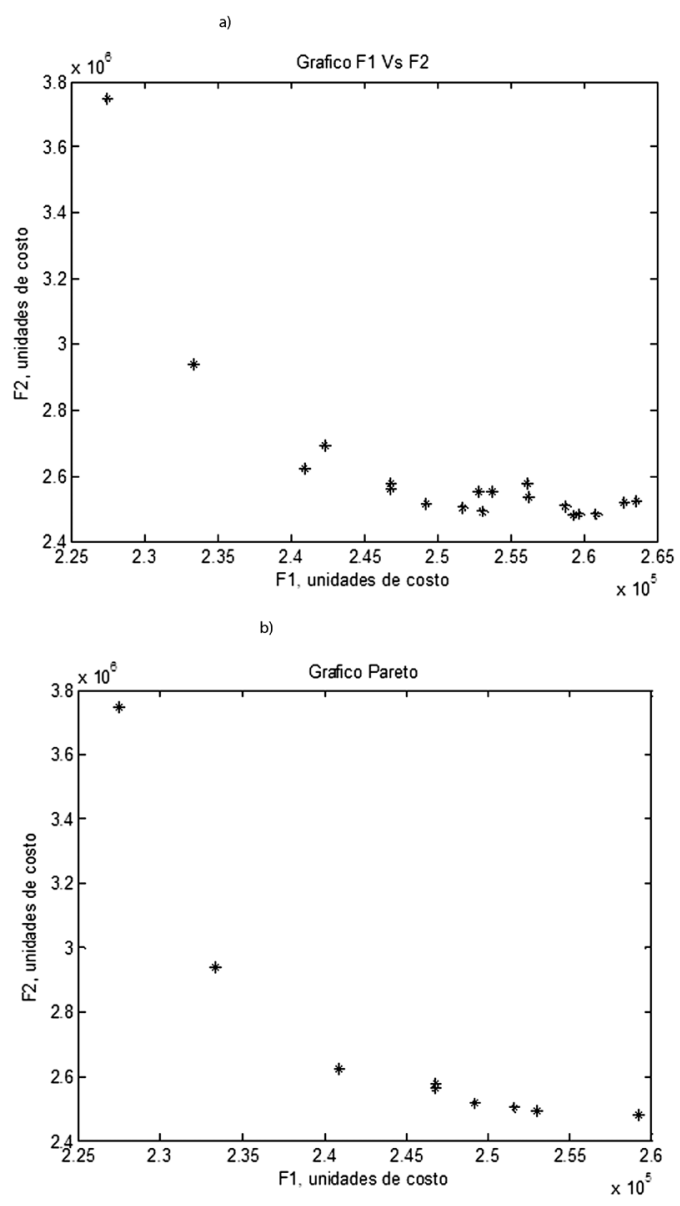

Figura 2. Graficas de resultados. a) Grafica de resultados instancia Xerox. b) Frente Pareto instancia Xerox.

Dado que se hace muy extenso y repetitivo la presentación de todos los resultados para todas las instancias con sus respectivos frentes de Pareto, se muestra en la tabla 4 el resultado con menor costo total $(\mathrm{FT}=\mathrm{F} 1+\mathrm{F} 2)$ para cada una de las instancias. Para la instancia Xerox, por ejemplo, este valor se encontró para un $\alpha=0$ con una $\mathrm{FT}=$ $2,737,679$.

Los resultados multiobjetivo indican que el modelo matemático representa y sirve para optimizar el costo de manejo de materiales y las relaciones de cercanía a través de la construcción de un frente de Pareto que proporciona variedad de resultados y cuya implementación depende de las preferencias o necesidades de la empresa por uno u otro objetivo. Para instancias en donde las estaciones son pocas (menos de 7) y donde las matrices de costos y de importancia tienen cierta semejanza los objetivos convergen hacia una misma solución.

A partir de los resultados se puede establecer también que los objetivos de optimización de costo de manejo de materiales y optimización de relaciones de cercanía no son necesariamente contradictorios y que en ocasiones ambos conducen a distribuciones semejantes. Se debe tener cuidado al momento de la determinación de la matriz de relaciones de cercanía o ratios de proximidad, cuidando que estos ratios no se establezcan teniendo en cuenta los flujos sino otros criterios.

\section{Conclusiones}

En el presente artículo, se propuso un modelo matemático para representar el problema de distribución de instalaciones de dimensiones fijas y áreas desiguales. Fueron utilizadas instancias de literatura para realizar la validación del problema. De las siete instancias utilizadas para la validación, en todas se obtuvo como resultado que el modelo propuesto es capaz de encontrar las soluciones óptimas.

El modelo matemático propuesto es eficaz ya que sirve para optimizar el costo de manejo de materiales y las relaciones de cercanía entre instalaciones. Además es eficiente al optimizar 12 de las 21 instancias utilizadas y encontrar respuestas de buena calidad desde el punto de vista de los costos de manejo de materiales y de las relaciones de cercanía para el resto de las instancias, proporcionando distribuciones prácticas desde el punto de vista la implementación.

La solución del problema de distribución de instalaciones a través del modelo propuesto proporciona a las organizaciones un frente de Pareto que permite la elección de la solución que más se ajuste a las necesidades de la organización. Los objetivos tratados para el problema de distribución en planta en esta investigación no son necesariamente contradictorios. Como se pudo observar en algunos resultados, la optimización del 
Tabla 4: Mejor resultado de la función total de costo.

\begin{tabular}{cccccc}
\hline Instancia & $\begin{array}{c}\text { Número De } \\
\text { Departamentos }\end{array}$ & $\boldsymbol{A}$ & $\boldsymbol{F 1}$ & $\boldsymbol{F 2}$ & $\boldsymbol{F 3}$ \\
\hline Apte & 9 & $0.30-0.90$ & $308.536,00$ & $2.881 .010,00$ & $3.189 .546,00$ \\
Xerox & 10 & 0.00 & $259.224,00$ & $2.478 .455,00$ & $2.737 .679,00$ \\
Hp & 11 & 0.00 & $162.484,00$ & $1.492 .749,99$ & $1.655 .233,99$ \\
Ami33 & 33 & 0.25 & $730.338,00$ & $13.797 .490,00$ & $14.527 .828,00$ \\
Ami49 & 49 & 0,10 & $8.788 .640,00$ & $174.844 .320,00$ & $183.632 .960,00$ \\
Example 1 & 7 & $0.50-0.75$ & 10633,999 & 5662,5 & 16296,499 \\
Example 2 & 12 & $0.35-0.60$ & $17.590,00$ & $4.900,00$ & $22.490,00$ \\
Example 3 & 18 & 0.4 & $52.522,50$ & $75.730,00$ & $128.252,50$ \\
Example 4 & 36 & 0.05 & $13.561,42$ & $522.472,50$ & $536.033,92$ \\
Nugent 5 & 5 & $0.05-1$ & 50,00 & 160,00 & 210,00 \\
Nugent 6 & 6 & $0.05-0.95$ & 86,00 & 240,00 & 326,00 \\
Nugent 7 & 7 & $0.00-0.60$ & 7,13 & 156,00 & 980,00 \\
Nugent 8 & 8 & $0.30-0.85$ & 234,00 & $1.090,00$ & $1.324,00$ \\
Nugent 12 & 12 & $0.00,0.10$ & 740,00 & $3.850,00$ & $4.590,00$ \\
Nugent 15 & 15 & 0,60 & $1.476,00$ & $7.230,00$ & $8.706,00$ \\
Nugent 20 & 20 & 0,20 & $3.300,00$ & $17.950,00$ & $21.250,00$ \\
Nugent 30 & 30 & 0,00 & $7.750,00$ & $54.660,00$ & $62.410,00$ \\
\hline
\end{tabular}

costo de manejo de materiales y de las relaciones de cercanía no es siempre contradictoria, ya que en algunos casos la solución óptima de cada uno de los objetivos converge hacia una misma distribución. El hecho de los objetivos no ser contradictorios totalmente en muchos de los sistemas productivos, torna en algunos casos innecesaria la consideración del modelo como multiobjetio y pude ser la razón de que pocos autores hasta la fecha hayan intentado abordar el problema de FLP desde esta perspectiva.

Para problemas de gran tamaño $(\mathrm{n}>12$ en esta investigación) el modelo no encuentra soluciones optimas en menos de 10.000 segundos por lo que se recomienda la utilización de técnicas heurísticas y/o meta-heurísticas para encontrar buenas soluciones en este tipo de problemas.

Como futuros trabajos a partir de esta investigación se puede realizar la implementación del modelo propuesto en un sistema de manufactura real en el que se pueda determinar los costos, los flujos y las relaciones de cercanía entre instalaciones. También es posible utilizar el enfoque multiobjetivo en otros tipos de distribución de instalaciones diferentes al problema de dimensiones fijas y áreas desiguales y aplicar técnicas heurísticas y metaheurísticas para mejorar la calidad de respuesta y el tiempo de cálculo empleado para resolver el problema multiobjetivo que representa el modelo propuesto. Es recomendado, por último, el diseño de metodologías o herramientas que permitan determinar los ratios de relaciones de cercanía entre instalaciones independientemente del flujo de producción que existente.

\section{Referencias bibliográficas}

Chiang, W.-C. (2001). Visual facility layout design system. International Journal of Production Research 39 (9), 1811-1836.

Drira, A., Pierreval, H., \& Hajri-Gabouj, S. (2007). Facility layout problems: A survey. Annual Reviews in Control 31(2), 255-267.

Garci, L., Pierreval, H., Salas-Morera, L., \& Arauzo-Azofra, a. (2010). An Interactive Genetic Algorithm with c-Means clustering for the Unequal Area Facility Layout Problem. 2010 10th International Conference on Intelligent Systems Design and Applications, p. 61-66. 
Kundu, A., \& Dan, P. K. (2012). Metaheuristic in facility layout problems: current trend and future direction. International Journal of Industrial and Systems Engineering 10(2), 238.

Maniya, K. D., \& Bhatt, M. G. (2011). An alternative multiple attribute decision making methodology for solving optimal facility layout design selection problems. Computers \& Industrial Engineering 61(3), 542-549.

Nugent, C. E., Vollmann, T. E., \& Ruml, J. (1968). An Experimental Comparison of Techniques for the Assignment of Facilities to Locations. Operations Research 16(1), 150-173.

Ohmori, S., Yoshimoto, K., \& Ogawa, K. (2010). Solving Facility Layout Problem via Particle Swarm Optimization. In Computational Science and Optimization CSO 2010 Third International Joint Conference on IEEE Computer Society Vol. 1, p. 409-413.

Papageorgiou, L. G., \& Rotstein, G. E. (1998). Continuous-Domain Mathematical Models for Optimal Process Plant Layout. Industrial \& Engineering Chemistry Research 37(9), 3631-3639.
Sadrzadeh, A. (2012). A genetic algorithm with the heuristic procedure to solve the multi-line layout problem. Computers \& Industrial Engineering 62(4), 1055-1064.

Salas-Morera, L., Garcia-Hernandez, L., \& Arauzo-Azofra, A. (2011). An evolutionary algorithm for the unequal area facility layout problem. International Conference on Intelligent Systems Design and Applications (ISDA). IEEE, p. 414-419.

Shah, K., Ripon, N., Glette, K., Høvin, M., \& Tørresen, J. (2010). Dynamic Facility Layout Problem with Hybrid Genetic Algorithm. International Conference on Cybernetic Intelligent Systems. IEEE, p. 1-6.

Tompkins, J. A., \& White, J. A. (1984). Facilities planning. J. Wiley, Ed. Third Edit., p. 734. New York.

Xu, G., \& Papageorgiou, L. G. (2009). Process plant layout using an improvement-type algorithm. Chemical Engineering Research and Design 87(6), 780-788. 